\title{
On the strain-rate sensitivity of columnar ice
}

\author{
M. E. Manley, E. M. Schulson \\ Thayer School of Engineering, Dartmouth College, Hanover, New Hampshire 03755, U.S.A.
}

\begin{abstract}
A power law relation between stress and strain rate of the form $\sigma \propto \dot{\varepsilon}^{1 / n}$ was used to describe the response to strain rate of Sl ice loaded across the columns at $-10^{\circ} \mathrm{C}$. The rate exponent, $n$, decreased with increasing strain from about 4.6 at an observed peak on the load displacement curve to approximately 2.6 at a shortening of $2 \%$. Analysis of these results and of the results of other authors on different forms of ice deformed at the same temperature suggests that the power law exponent, $n$, is proportional to $F_{\mathrm{c}} / F_{\mathrm{g}}$. The parameter $F_{\mathrm{c}} / F_{\mathrm{g}}$ is the far-field basal dislocation climb force divided by the glide force.
\end{abstract}

During an investigation of the ductile compressive failure of S1 ice (designation of Michel and Ramseier, 1971), we performed a series of experiments to determine the strain-rate sensitivity, $n$, of the flow stress $\sigma=B \dot{\varepsilon}^{1 / n}$ where $B$ is a constant and $\dot{\varepsilon}$ is the strain rate. Instead of obtaining one value of about $n=3$ as one might expect from Glen's law for power law creep of polycrystals (Duval and others, 1983; Weertman, 1983), we obtained several values whose range seemed too large to ascribe to experimental scatter. This result was reminiscent of earlier observations by Frederking (1977) and by Schulson and Buck (1995). It also brought to mind Weertman's (1983) compilation. In an attempt to rationalize this behavior, we found a relationship between the strain-rate sensitivity and the ratio of the climb force to the glide force on basal dislocations. Hence this note.

The S1 ice was grown unidirectionally as a sheet from fresh water. The procedure and the material are fully described elsewhere (Manley, 1996). The ice consisted of columnar-shaped grains about $8 \mathrm{~mm}$ in diameter whose crystallographic $c$ axes were parallel to the long axes of the columns to within $\pm 5^{\circ}$. Plate-like specimens $(159 \mathrm{~mm} \times$ $159 \mathrm{~mm} \times 25 \mathrm{~mm}$ ) were milled from the sheet, with the columns perpendicular to the large faces. Five specimens were then compressed uniaxially across the columns (i.e. along a direction more or less within the basal plane) using a servohydraulic loading frame, at a temperature of $-10 \pm 0.2^{\circ} \mathrm{C}$. Each specimen was initially strained at a constant but different rate $\left(\dot{L} / L_{0}=10^{-6} \mathrm{~s}^{-1}, 3 \times 10^{-6} \mathrm{~s}^{-1}, 10^{-5} \mathrm{~s}^{-1}, 5 \times 10^{-5} \mathrm{~s}^{-1}\right.$ and $\left.10^{-4} \mathrm{~s}^{-1}\right)$ to beyond the peak in the stress-strain curve; i.e. to a strain of about 0.005 as measured using a displacement gauge mounted across the ice. The peak occurred at a strain of about 0.003. Subsequently, and without unloading, the strain rate was either instantaneously increased or decreased by a factor of ten and the ice was shortened an additional $0.2-0.4 \%$, after which the strain rate was instantaneously restored to its initial value and the ice was shortened by this amount again. This cycle was repeated twice before the specimen was unloaded. The strain-rate sensitivity was computed from the peak in the flow stress in the usual manner (i.e. from the slope of a plot of $\log$ flow stress vs log strain rate) and from the flow stresses just before $\left(\sigma_{1}\right)$ and just after $\left(\sigma_{2}\right)$ the change in strain rate through the relationship $n=\log \left(\dot{L}_{2} / \dot{L}_{1}\right) / \log \left(\sigma_{2} / \sigma_{1}\right)$.

The ice kinked in every experiment, owing to the load being applied in the basal plane. An analysis is given elsewhere (Manley and Schulson, 1997). This feature, however, did not prevent the rate sensitivity from being determined. The stress-strain curves are given by Manley (1996). Upon analysis, they showed that the sensitivity decreased from $n=4.6$ at an axial strain of 0.003 to $n=2.6$ at a strain of 0.02 (Fig, 1). The vertical bars denote the spread in values obtained from the different specimens.

To rationalize this dependence, we assumed that the ice deformed by both the glide of dislocations in the basal plane and the climb of dislocations out of the basal plane, following Duval and others (1983) and Weertman (1983). We then calculated the ratio of the climb force, $F_{\mathrm{c}}$, to the glide force, $F_{\mathrm{g}}$, on the dislocations within the kink band, by knowing the lattice rotation from the optical analysis given elsewhere

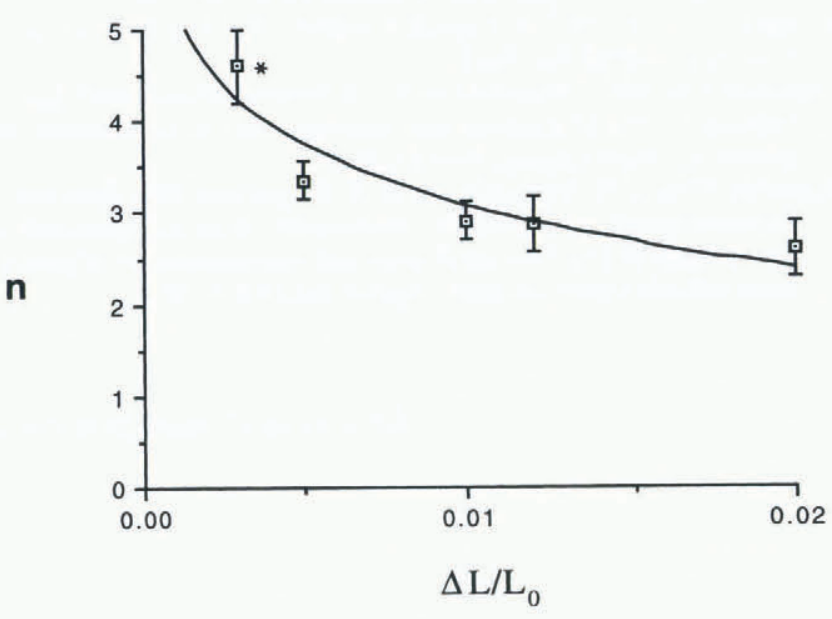

Fig. 1. The exponent, $n$, from the relation $\sigma \propto \dot{\varepsilon}^{1 / n}$ vs strain, for S1 fresh-water ice deformed at $-10^{\circ} \mathrm{C}$. The star (*) was obtained from a series of six tests (Manley, 1996) from $10^{-6}$ to $10^{-4} s^{-1}$ where the log of the peak compressive stress was plotted vs the log of the strain rate. All of the other data were obtained from strain-rate change tests. 
(Manley and Schulson, 1997) and by making the transformation given in the Appendix. We found that $n$ increased more or less linearly with the ratio $F_{\mathrm{c}} / F_{\mathrm{g}}$ (Fig. 2a). We made

n

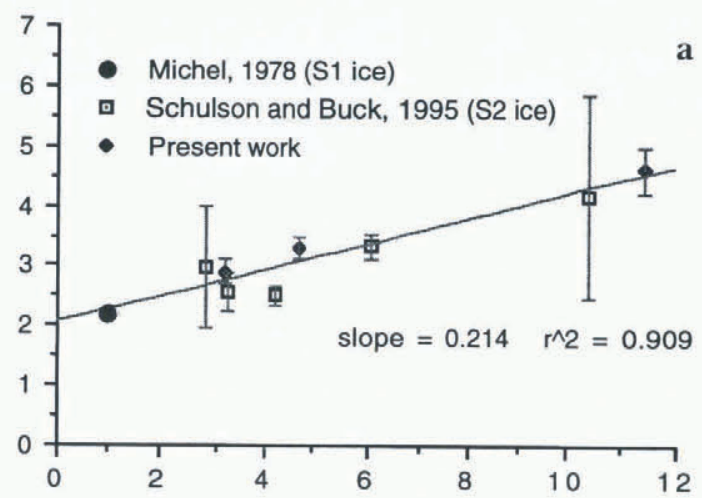

$\mathrm{F}_{\mathrm{c}} / \mathrm{F}_{\mathrm{g}}$

(a)

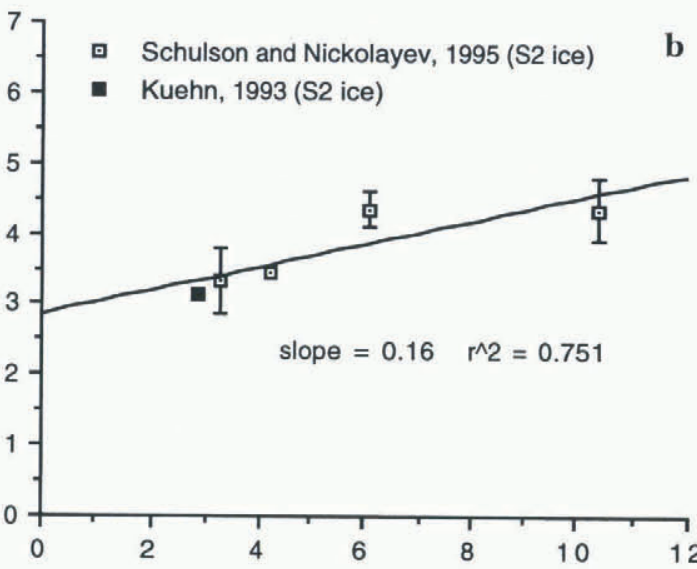

Fig. 2. Plots of $n$ values at $-10^{\circ} \mathrm{C}$ vs $F_{\mathrm{c}} / F_{\mathrm{g}}$ for (a) freshwater columnar ice and (b) saline columnar ice. Although five points are shown from the present work in Figure 1, only three points are shown here, because we determined the kink reorientation for the S1 ice in only three cases (Manley and Schulson, 1997).

a similar calculation for $\mathrm{S} 2$ fresh-water ice (where the $c$ axes are randomly oriented within a plane more or less perpendicular to the long axis of the columnar grains (Michel and Ramseier, 1971; Michel, 1978) deformed at $-10^{\circ} \mathrm{C}$ by compressing biaxially across the long axis of the columns (Schulson and Buck, 1995), as shown in the Appendix (Fig. 5). The results fit the same curve (Fig. 2a). Finally, we made the analysis for S2 saline ice of 4-5 ppt salinity, also loaded biaxailly across the columns at $-10^{\circ} \mathrm{C}$ (Kuehn, 1993; Schulson and Nickolayev, 1995). Again, $n$ increased more or less linearly with increasing $F_{\mathrm{c}} / F_{\mathrm{g}}$ (Fig. 2b).

Thus, it appears that to a first approximation $n=n_{\mathrm{o}}+$ $k F_{\mathrm{c}} / F_{\mathrm{g}}$ where $n_{\mathrm{o}}$ is about 2 and 2.8 for fresh-water and saltwater ice, respectively, and $k$ is around 0.2 for both materials at $-10^{\circ} \mathrm{C}$.

We suggest that this relationship reflects a transition from essentially two-dimensional (glide) to three-dimensional (glide plus climb) dislocation motion as $n$ increases from 2 to 3 . The higher $n$ values may reflect a non-linear dependence of dislocation velocity on stress. It is interesting to note, however, that our criterion predicts that $n=2.2$ for uniaxially loaded single crystals whose basal planes are inclined $45^{\circ}$ to the direction of loading. This is in agreement with the value $n=2.1$ obtained by Homer and Glen (1978) who crept such crystals at $-10^{\circ} \mathrm{C}$.

Hopefully, this note will stimulate further discussion.

\section{ACKNOWLEDGEMENTS}

We would like to thank E. Gratz andJ. Melton for their help with laboratory equipment. Also, we acknowledge the helpful comments by the anonymous reviewers. The work was supported by the U.S. Army Research Office, grant No. DAAL03-92-G-0187.

\section{REFERENCES}

Duval, P., M. F. Ashby and I. Anderman. 1983. Rate-controlling processes in the creep of polycrystalline ice. 7 . Phys. Chem., 87 (21), 4066-4074.

Frederking, R. 1977. Plane-strain compressive strength of columnar-grained and granular-snow ice. 7. Glaciol., 18 (80), 505-516.

Homer, D. R. and J.W. Glen. 1978. The creep activation energies of ice. $\mathcal{F}$. Glaciol., $21(85), 429-444$.

Kuehn, G. A. 1993. Laboratory-grown saline ice - a first-year sea ice ana$\log$ : the uniaxial compressive properties and the uniaxial tensile properties after compressive pre-strain. (M.S. thesis, Dartmouth College, Hanover, NH.

Manley, M. E. 1996. Deformation in S1 and S2 ice loaded across the columns. (M.Sc, thesis, Dartmouth College, Hanover, NH.)

Manley, M. E. and E. M. Schulson. 1997. Kinks and cracks in Sl ice under across-column compression. Philos. Mag. Lett., 75 (2), 83-90.

Michel, B. 1978. Ice mechanics. Québec, Qué., Presses de L'Université Laval.

Michel, B. and R. O. Ramseier. 1971. Classification of river and lake ice. Can. Geotech. J., 8 1), $36 \quad 45$.

Schulson, E. M. and S. E. Buck. 1995. The ductile-to-brittle transition and ductile failure envelopes of orthotropic ice under biaxial compression. Acta Metall. Materialia, 43 (10), 3661-3668.

Schulson, E. M. and O.Y. Nickolavev. 1995. Failure of columnar saline ice under biaxial compression: failure envelopes and the brittle-to-ductile transition. 7. Geophys. Res., 100 (BI1), 22,383-22,400. Correction: f. Geophys. Res., 101 (B3), 1996, 5659.

Weertman, J. 1983. Creep deformation of ice. Annu. Rev. Earth Planet. Sci., 11, $215-240$.

\section{APPENDIX}

\section{GALGULATIONS OF $\boldsymbol{F}_{\mathrm{c}} / \boldsymbol{F}_{\mathrm{g}}$}

Consider an edge dislocation within a volume element (Fig. 3). The climbing force is related to the normal stress by $F_{\mathrm{c}}=b \sigma_{\mathrm{n}}$, where $b$ is the Burger's vector. The glide force is related to the shear stress by $F_{\mathrm{g}}=b \sigma_{\mathrm{s}}$. So

$$
\frac{F_{\mathrm{c}}}{F_{\mathrm{g}}}=\frac{\sigma_{\mathrm{n}}}{\sigma_{\mathrm{s}}} .
$$

\section{Calculation for S1 ice loaded uniaxially}

Assuming a uniform applied stress (Fig. 4), the stresses on a volume element containing a basal edge dislocation on the $x_{1}^{\prime}-x_{2}^{\prime}$ plane are

$$
\sigma_{\mathrm{n}}=\sigma_{11}^{\prime}=\frac{1}{2} \sigma(1+\cos 2 \theta)
$$

and

$$
\sigma_{\mathrm{s}}=\sigma_{31}^{\prime}=\frac{1}{2} \sigma \sin 2 \theta,
$$

where $\theta$ is the tilting angle of the basal planes within the 


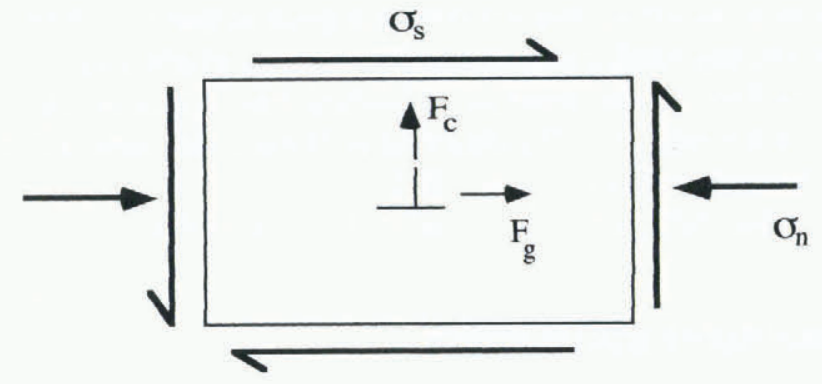

Fig. 3. Sketch of glide and climb forces on an edge dislocation.

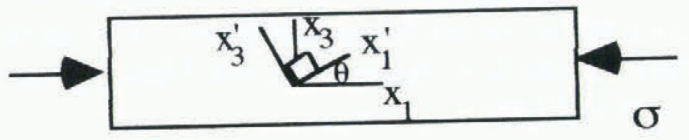

Fig. 4. Sketch showing reorientation of basal slip plane within kink.

kink band (Manley and Schulson, 1997). Using Equation $(\mathrm{Al})$, the ratio is

$$
\frac{F_{\mathrm{c}}}{F_{\mathrm{g}}}=\left(\frac{1+\cos 2 \theta}{\sin 2 \theta}\right) .
$$

The ratio decreases as $\theta$ increases from near zero to $\pi / 4$. The ratio approaches infinity for $\theta=0$. At this point basal glide cannot occur.

\section{Calculation for S2 ice loaded biaxially}

For this case the basal planes (shown as lines crossing the columns; Fig. 5) of individual columns are oriented ran-

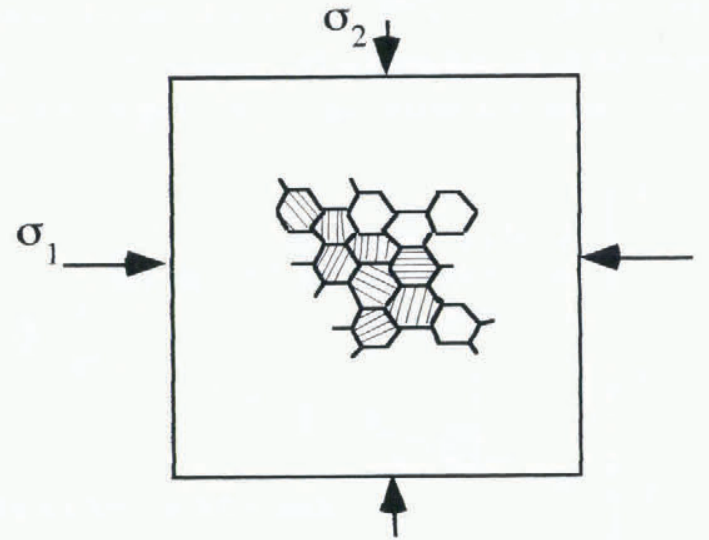

Fig. 5. Sketch showing across-column biaxial loading of $S 2$ ice. Traces of the basal planes are shown.

domly with respect to the principal stresses (the columns are pointing out of the page in the figure). For an individual column having its basal plane oriented at some angle $\alpha$ with respect to the maximum principal stress axis $\left(\sigma_{1}\right)$ the ratio is

$$
\frac{F_{\mathrm{c}}}{F_{\mathrm{g}}}=\left(\frac{\cot \alpha+R_{21} \tan \alpha}{1-R_{21}}\right) \text { where } R_{21}=\sigma_{2} / \sigma_{1} .
$$

For a given angle $(>0)$ the function increases as $R_{21}$ increases to near unity. The ratio approaches infinity at $\alpha=0, \pi / 2$ and also when $R_{21}=1$. A global ratio for a given specimen was found by assuming the average angle is $\alpha=19.5^{\circ}$. A single crystal placed at this angle with respect to the loading direction would have the same average Schmidt factor as the average factor $(0.32)$ for S2 ice. 